\title{
Identification of the Effect of Depression on Risky Sexual Behavior: Exploiting a Natural Experiment
}

\author{
Susan L. Averett \\ averetts@lafayette.edu \\ Yang Wang \\ wangy@lafayettte.edu \\ Department of Economics \\ Lafayette College \\ Easton, PA 18042
}

November 10, 2011

We thank Dhaval Dave and Edward Gamber for thoughtful comments on an earlier draft. Any remaining errors are our own.

The research uses data from Add Health, a program project designed by J. Richard Udry, Peter S. Bearman, and Kathleen Mullan Harris, and funded by a grant P01-HD31921 from the Eunice Kennedy Shriver National Institute of Child Health and Human Development, with cooperative funding from 17 other agencies. Special acknowledgment is due Ronald Rindfuss and Barbara Entwisle for assistance in the original design. Persons interested in obtaining Data Files from Add Health should contact Add Health, The University of North Carolina at Chapel Hill, Carolina Population Center, 123 W. Franklin Street, Chapel Hill, NC 27516-2524

(addhealth@unc.edu). No direct support was received from grant P01-HD31921 for this analysis. 


\section{Introduction:}

Depression is pervasive in the U.S. Recent data from the CDC's Behavioral Risk Factor Surveillance Survey indicated that in 2008-09, 9 percent of respondents met the criteria for current depression and 3.4 percent met the criteria for major depression (CDC, 2010a). That same data indicated that women are more likely to report major depression (4.0\% versus $2.7 \%$ ). The economic costs of depression are substantial. Greenberg et al. (2003), using a human capital approach, estimated the economic costs of depression to be \$83.1 billion in 2000 with \$26.1 billion (31\%) of direct medical costs, $\$ 5.4$ billion (7\%) of suicide-related mortality costs, and $\$ 51.5$ billion (62\%) of workplace costs. One cost that has received little attention in the economics literature is the potential for depression to cause an individual to engage in risky sexual behavior. Depression may lead to risky sexual behavior as it may impair cognitive function and memory, decrease one's ability to control impulsive behavior, increase emotional reactivity in peer relationships, reduce motivation, and increase fatalism. These effects of depression may inhibit an individual's clear perception of the risk of contracting an STD and their ability to prevent risky sexual behavior (Khan et al., 2009).

Despite a substantial body of research that links depression and risky sexual behavior for both males and females across a broad age range, definitively establishing the direction of causality remains elusive. Several studies present strong evidence that depression and risky sexual behaviors are positively correlated using cross sectional data (see Shrier et al., 2001 and cites within). However, such studies are not indicative of causal relationships due to the potential endogeneity of depression. For example, those who engaged in sexual intercourse without a condom may become depressed and those who have an STD may also become depressed. In fact, there is a body of research that posits that risky sexual behavior itself is a cause of depression, even when the sexual behavior is not forced (Ethier et al., 2006). 
Research in the medical and adolescent health fields has tried to establish the direction of causality from depression to risky sexual behaviors using the temporal ordering of events. Much of this research suggests that depressed adolescents and young adults are more likely to engage in risky sexual behaviors (Harris, et al., 2002; Longmore et al., 2004; Hallfors et al., 2005; Mazzaferro et al., 2006; Mota et al., 2010 and Kahn et al., 2009), have an increased risk of a sexually transmitted infection (Shrier et al., (2009)), and are less likely to use condoms or use them appropriately (Shrier et al., 2011; Brown et al., 2006; Lehrer et al., 2006; Morrison et al., 2008). Yet even this research, which uses panel instead of cross-sectional data, does not definitively establish causality because there remains the possibility that a third unobservable factor could be correlated with the earlier episode of depression and the current risky sexual behavior.

In this paper, we exploit a unique opportunity in our empirical setting to identify the causal effect of depression, measured by the Center for Epidemiologic Studies Depression Scale (CESD), on a young adult’s propensity to engage in risky sexual behaviors. Specifically, an unexpected event -the September 11 attacks (9/11)-occurred, during the collection Wave III (July 2001 to April 2002) of the AddHealth. Because of its unexpected nature and minimal impact on the continuation of Add Health interviews, 9/11 can be viewed as a natural experiment which randomly assigned young adults interviewed before 9/11 into the "control" group and those after the "treatment" group. ${ }^{1}$ In our data, we find that this "treatment" leads to an exogenous and significant variation in the levels of depression between individuals in the "control" vs.

\footnotetext{
${ }^{1}$ Wang and Yang (2010) also explore the exogeneity of 9/11 in their analysis of effects of depression on weight-related behaviors using the same data set. They use a different sample, with a different measure of depression.
} 
“treatment” group: the latter experienced a significant increase in CESD scores. ${ }^{2}$ Our results show that depression does lead to more engagement in risky sexual behavior.

\section{Data}

Our data are from the AddHealth which is a school-based longitudinal study of a nationally representative sample of adolescents in grades 7 to 12 in the United States during the 1994-5 school year. ${ }^{3}$ We use Wave III of the AddHealth for our study with respondents having been interviewed from July 2001 to April 2002. We focus our analysis on women since we established that depression is more prevalent in women and women bear a large proportion of the costs of risky sexual activity ${ }^{4}$. We also limit our sample to unmarried women because the decisions surrounding sexual activity are likely to be different for married versus unmarried women.

Nine questions asked in Wave III are used to generate a CESD score to measure a young women's level of depression: How often did you feel this way during the past week? (1) You were bothered by things that usually don't bother you; (2) You felt that you could not shake off the blues, even with help from your family and your friends; (3) You felt that you were just as good as other people; (4) You had trouble keeping your mind on what you were doing; (5) You felt depressed; (6) You were too tired to do things, during the past seven days; (7) You enjoyed

\footnotetext{
${ }^{2}$ There is evidence that among disaster workers, 9/11 was associated with depression and Acute Stress Disorder which severely impaired their functioning (Biggs et al., 2010).

${ }^{3}$ More details on this dataset are available at http://www.cpc.unc.edu/projects/addhealth/design/designfacts.

${ }^{4}$ Females bear the brunt of most of the health consequences of risky sexual activities. One in four young women is infected with at least one of the most common STDs (AGI, 2009). STDs (other than HIV/AIDS) may have more life-threatening consequences for women (e.g. Pelvic Inflammatory Disease, ectopic pregnancy and cervical cancer) than for men. (Hillis and Wasserheit, 1996; Koshiol et al., 2008). The costs of the nine million new cases of the most prevalent STDs that occurred among 15 to 24 year olds in 2000 amounted to \$65 billion (Chesson et al., 2004).
} 
life; (8) You felt sad; (9) You felt that people disliked you. For each question, respondents chose from the following numbers as their answers: 0 : never or rarely; 1 : sometimes; 2 : a lot of the time; 3: most of the time or all of the time. Combining all nine questions, we obtain a CESD score, ranging from 0 (least likely to be depressed) to 27 (most likely to be depressed). The CESD score is a common measure of depressive symptoms (Radloff, 1977). Because this score is typically constructed using 20 questions including the nine described above and because it is usually used as a screening test than a diagnosis for depression, we directly use the total scores (from nine questions) in our empirical analysis instead of (arbitrarily) assigning our samples into depressed vs. non-depressed groups.

Our (binary) outcome variables measuring risky sexual behaviors of single young women in a romantic relationship include: a) whether the young woman is sexually active, b) whether she engages in oral sex, c) whether she engages in anal sex, and d) whether she uses condoms during sex. ${ }^{5}$ Previous research has shown the importance of examining not just vaginal intercourse but also other sexual behaviors among young adults (Averett et al., forthcoming). In our analysis, we also control for these single young women's age, race, ethnicity, years of education, self-rated health status, drinking and smoking behaviors and marijuana use, selfimage, and attitudes towards risk or impulsivity.

Because Wave III of Add Health started gradually in July 2001 (with a few exceptions), just two months before 9/11, in order to construct comparable "control" and "treatment" groups, we focus on the young women interviewed within one month before and after 9/11. We find that

\footnotetext{
${ }^{5}$ Having multiple sex partners could be another risky sexual behavior with substantial health consequences. However, in Wave III AddHealth asked women only about sex within the context of a relationship not the number of concurrent sexual partners.
} 
there were approximately the same number of interviews one month before and one month after 9/11, consistent with what AddHealth described as an un-interrupted interview schedule.

Respondents for Wave III are asked to compile a relationship roster where they selfidentify all romantic relationships they have had since 1995 (wave I of the AddHealth).They also asked if the relationship was currently occurring at the time of the interview. We include in our sample only those respondents currently in relationships at the time of interview because of the unique identification method we use - the attacks of September 11. If the relationship was too far in the past we would not be identifying the effect of depression on sexual activity accurately given the temporal nature upon which our identification scheme rests. Because the questions about sexual activity in Wave III were asked only of women in relationships, we cannot explore whether or not depression caused these women to engage in sexual activity with someone they were not in a romantic relationship with or to engage in sexual activity with strangers.

Our full sample consists of 4,151 women aged 18 to 26 years. Most of our respondents are White (68\%) or African American (25\%). The average years of education are around 13 years and a half, and the average age is 21 . Thirty-five percent smoked for at least 30 days, and during the year before interview $72 \%$ of these women drank alcohol, and 31\% used marijuana. The average self-rated health is about 2.05 , which is slightly worse than 'very good'. The mean CESD score for the 9 questions asked in the Wave III Add Health for our sample is 8.12. As for the outcome variables, more than $95 \%$ of these women are sexually active in their relationships. $67 \%$ and $11 \%$ of them engage in oral and anal sex, respectively. Fewer than $2 \%$ of the women in our sample report using condoms.

\section{Econometric Model}

The following econometric model is brought to our data: 


$$
y_{i}=\beta_{0}+\beta_{1} \mathrm{CESD}_{\mathrm{i}}+x_{i}^{\prime} \beta_{2}+\varepsilon_{i}
$$

where is $\varepsilon_{i}$ the disturbance term for individual $i, \mathrm{CESD}_{i}$ is her CESD score which could be potentially endogenous, $x_{i}$ is a vector of her characteristics, and $y_{i}$, as we mentioned in the Data section, includes 4 binary outcomes for each respondents: (1) whether the young woman is sexually active, (2)whether she engages in oral sex, (3) whether she engages in anal sex, and (4) whether she uses condoms during sex. Our instrumental variable, not shown in Equation (1) but used in our 2SLS estimation, is a binary variable set to 1 if the young woman was interviewed on or after the occurrence of 9/11 and 0 otherwise. When we graph the weekly average CESD scores against interview dates, there is indeed a jump in the CESD scores right after 9/11 indicating that this instrumental variable is very likely to be strong; our "treatment" group is indeed treated in this "natural experiment". The strength of our instrument is also confirmed by our estimation results which we discuss below.

\section{Results}

Table 1 presents the OLS and 2SLS estimates of the effect of depressed mood on our four outcome variables. We show only the results from the model that includes the full set of controls discussed above although we do not show the coefficients from our control variables in this table due to space limitations. The OLS results reveal a positive and significant coefficient only on the probability of engaging in sexual intercourse. This finding that depression is positively related to sexual activity has been reported by others as summarized in our Introduction section.

Table 1: Coefficients on CESD score*

\begin{tabular}{|l|l|l|}
\hline Outcome & OLS & 2SLS \\
\hline Having Sex $(\mathbf{N}=4003)$ & $0.004^{* * *}$ & $\begin{array}{l}0.021^{*} \\
(\mathbf{0 . 0 1 1}) \\
{[17.42)}\end{array}$ \\
\hline Oral Sex $(\mathbf{N}=3751)$ & $(0.001)$ & $0.055^{* * *}$ \\
& -0.001 & $(0.025)$ \\
\hline
\end{tabular}




\begin{tabular}{|l|l|l|}
\hline & & {$[20.27]$} \\
\hline Anal Sex (N=3700) & -0.002 & 0.028 \\
& $(0.002)$ & $(0.018)$ \\
& & {$[19.60]$} \\
\hline Condom Use (N=3747) & -0.001 & $-0.019 * *$ \\
& $(0.001)$ & $(0.007)$ \\
& & {$[16.66]$} \\
\hline
\end{tabular}

*Robust standard errors in parentheses. First Stage F-statistics in square brackets. All models include controls for race, ethnicity, years of education, age, self-rated health, whether the respondent drinks, smokes or uses marijuana, self-image and attitudes towards risk.

Moving to the 2SLS estimates in column 2 of table 1, it is clear that our instrument is strong as indicated by the F-statistics (shown in square brackets) from the first stage all exceeding 10 (The complete set of all results are available in Averett and Yang, 2011). Specifically, our 2SLS results indicate that not only are young women with elevated depressed mood more likely to be sexually active, they are also more likely to engage in oral sex and to have sex without a condom, even after controlling for our rich set of covariates.

Our 2SLS results are larger than our OLS results for having suggesting that OLS underestimates the effect of depression on having sex. We ran two additional specifications as a check on the robustness of our results. First, we ran a model to determine if 9/11 was a statistically significant determinant of relationship status and we found that it was not. Finally, we also included the 9/11 variable in the fully specified OLS equation and found that it was never statistically significant. Thus, we feel confident that our instrument is legitimately excluded from the second stage.

\section{Conclusion}

In this paper we have added to the existing literature by exploiting the timing of 9/11 coincident with the data collection of Wave III of the AddHealth survey to identify the effect of depression on risky sexual activity among young adult women. We focus on contemporary measures of 
sexual activity such as anal and oral intercourse in addition to vaginal intercourse-these measures are particularly worthy of study because they are indicative of current sexual practices and they are often performed without barrier protection making them particularly risky. Given the odds and substantial costs of a young adult woman contracting an STD, it is also important to understand the risk factors underlying an STD diagnosis so policymakers can make appropriate policy recommendations.

Our findings indicate that higher CESD scores are significantly associated with having sex and with two risky sexual behaviors that are relatively common among young women (oral sex and failure to use a condom). These findings indicate that initiatives to make mental health care more available to a wider array of the population such as that proposed by President Obama (www.barackobama.com) may also have the benefit of reducing risky sexual activity. As of January 2010, only 22 states plus the District of Columbia mandated mental health care (Kaiser Foundation, 2010).

\section{References:}

AGI, 2009. Facts on Sexually Transmitted Infections in the United States (available at: http://www.guttmacher.org/pubs/FIB_STI_US.html). Last accessed 10/28/2011.

Aral SO, Patel DA, Holmes KK, Foxman B. Temporal trends in sexual behaviors and sexually transmitted disease history among 18- to 39-year-old Seattle, Washington, residents: results of random digit-dial surveys. Sexually Transmitted Diseases 2005; 32:710 -717.

Averett, Susan., Hope Corman and Nancy Reichman. 2011. "Effects of Overweight on Risky Sexual Behavior of Adolescent Girls" Forthcoming, Economic Inquiry.

Averett, Susan and Yang Wang. 2011. "Identification of the Effect of Depression on Risky Sexual Behavior: Exploiting a Natural Experiment” http://sites.lafayette.edu/averetts/

http//www.barackobama.com/pdf/issues/HealthCareFullPlan.pdf

Biggs, Q., C. Fullerton, J. Reeves, T. Grieger, D. Reissman, R.Ursano, 2010.“Acute Stress Disorder, Depression, and Tobacco Use in Disaster Workers Following 9/11” American Journal of Orthopsychiatry 80(4)586:592. 
Brown,Larry K., Marina Tolou-Shams, Celia Lescano, Christopher Houck, Jessica Zeidman, David Pugatch, Kevin J. Lourie and Project SHIELD Study Group, 2006. “Depressive Symptoms as a Predictor of Sexual Risk among African American Adolescents and Young Adults”, Journal of Adolescent Health, 39(3):444

CDC. 2010a. “Current Depression Among Adults-United States, 2006 and 2008” Journal of the American Medical Association.304(20):2233-2235.

Chesson, H., J. Blandford, T. Gift, G. Tao and K. Irwin. 2004. "The Estimated Direct Medical Cost of Sexually Transmitted Diseases Among American Youth, 2000” Perspectives on Sexual and Reproductive Health. 36(1):11-19.

Edwards S, Carne C. “Oral sex and the transmission of viral STIs”. Sexually Transmitted Infections 1998; 74:6-10.

Ethier, Kathleen A., Trace S. Kershaw, Jessica B. Lewis, Stephanie Milan, Linda M. Niccolai, Jeannette R. Ickovics, 2006. "Self-esteem, emotional distress and sexual behavior among adolescent females: Inter-relationships and temporal effects”, Journal of Adolescent Health, 38(3):268-274.

Greenberg, P. E., Kessler, R. C., Birnbaum, H. G., Leong, S. A., Lowe, S. W., Berglund, P. A., \& Covey-Lisle, P. K. (2003). The economic burden of depression in the United States: How did it change between 1990 and 2000? Journal of Clinical Psychiatry, 64(12), 1465-1476.

Hillis SD and Wasserheit JN. 1996. Screening for chlamydia - a key to the prevention of pelvic inflammatory disease. New England Journal of Medicine. 334(21):1399-1401.

Jenness, S.M., Begier, E.M., Neaigus, A., Murrill, C.S., Wendel, T., and H. Hagan. (2010). Unprotected Anal Intercourse and Sexually Transmitted Diseases in High-Risk Heterosexual Women. American Journal of Public Health. June 17, 2010. Epub ahead of print.

Kaiser Foundation.2010. http://www.statehealthfacts.org/comparereport.jsp?rep=1\&cat=7. Last accessed 2/17/2011.

Khan, Maria J.; Jay S. Kaufman; Brian Wells Pence; Bradley N. Gaynes; Adaora A. Adimora; Sharon S. Weir; William C. Miller 2009. "Depression, Sexually Transmitted Infection, and Sexual Risk Behavior Among Young Adults in the United States”Archives of Pediatric and Adolescent Medicine. 2009;163(7):644-652.

Koshiol, J, L. Lindsay, J Pimenta, C.Poole, D. Jenkins, and and J. Smith. 2008. "Persistent Human Papillomavirus Infection and Cervical Neoplasia: A Systematic Review and MetaAnalysis” American Journal of Epidemiology168(2): 123-137.

Landry, D.J., and W. Turnbull. (1997). Issues in Brief: Sexually Transmitted Diseases Hamper Development Efforts. Available at: http://www.guttmacher.org/pubs/ib_std.html 
Lehrer, Jocelyn A., Shrier, Lydia A., Gortmaker, Steven, Buka, Stephen 2006. "Depressive Symptoms as a Longitudinal Predictor of Sexual Risk Behaviors Among US Middle and High School Students" Pediatrics. 118: 189-200

Lescano, C., Houck, C.D., Brown, L.K., Doherty, G., DiClemente, R.J., Fernandez, M.I., Pugatch, D., Schlenger, W.E., and B.J. Silver. (2009). Correlates of Heterosexual Anal Intercourse Among At-Risk Adolescents and Young Adults. American Journal of Public Health 99(6): 1131-1136.

Mazzaferro, Kathryn E., Pamela J. Murray, Roberta B. Ness, Debra C. Bass, Nadra Tyus, Robert L. Cook, 2006. "Depression, Stress, and Social Support as Predictors of High-Risk Sexual Behaviors and STIs in Young Women”, Journal of Adolescent Health, 39(4): 601-603

Mota, Natalie, Cox, Brian, Katz, Laurence, Sareen, Jitender 2010. "Relationship Between Mental Disorders/Suicidality and Three Sexual Behaviors: Results from the National Comorbidity Survey Replication” Archives of Sexual Behavior. 39(3):724-734

Shrier, Lydia, Sion Kim Harris, Maya Sternberg, William R. Beardslee, 2001. "Associations of Depression, Self-Esteem, and Substance Use with Sexual Risk among Adolescents”, Preventive Medicine, 33(3)179-189.

Shrier,Lydia Julia A. Schillinger, Parul Aneja, Peter A. Rice, Byron E. Batteiger, Phillip G. Braslins, Donald P. Orr, J. Dennis Fortenberry,2009. "Depressive Symptoms and Sexual Risk Behavior in Young, Chlamydia-Infected, Heterosexual Dyads", Journal of Adolescent Health, 45(1):63-69.

Shrier, Lydia, Courtney Walls, Christopher Lops, Henry A. Feldman, 2011. ”Correlates of Incorrect Condom Use among Depressed Young Women: An Event-level Analysis", Journal of Pediatric and Adolescent Gynecology, 24(1): 10-14.

Wang, Yang and Muzhe Yang. 2010. "Impacts of Depressed Mood on Physical Activity, Dietary Intake, and Weight Gain among Young Adults.” Working Paper. 